\title{
CARACTERIZAÇÃO DE PACIENTES SOB CUIDADOS PALIATIVOS SUBMETIDOS À PUNÇÃO VENOSA PERIFÉRICA E À HIPODERMÓCLISE
}

\section{CHARACTERIZATION OF PATIENTS UNDER PALLIATIVE CARE SUBMITTED TO PERIPHERAL VENIPUNCTURE AND HYPODERMOCLYSIS}

\section{CARACTERIZACIÓN DE PACIENTES EN ATENCIÓN PALIATIVA SOMETIDOS A UNA PUNCIÓN VENOSA PERIFÉRICA E HIPODERMOCLISIS}

Michele Rocha Moreira ${ }^{1}$, Ana Carolina de Souza르, Jéssica Villar ${ }^{3}$, Juliana Dias Reis Pessalacia ${ }^{4}$, Angelina Lettiere Viana ${ }^{5}$, Fabiana Bolela ${ }^{6}$

\begin{abstract}
RESUMO
Objetivo: caracterizar os pacientes oncológicos internados sob cuidados paliativos submetidos à punção venosa periférica e a hipodermóclise, segundo as variáveis sociodemográficas e clínicas. Método: estudo observacional, descritivo e prospectivo. Foram realizadas entrevistas com o paciente e/ou seu cuidador, consulta ao prontuário, avaliação e acompanhamento diário da punção. Resultados: participaram do estudo 45 pacientes de ambos os sexos e com idade superior a 18 anos. A avaliação funcional dos pacientes, no momento da internação, foi de $30 \%$, indicando pacientes extremamente incapacitados e com necessidade de hospitalização. Os sintomas mais frequentes apresentados pelos pacientes no dia da internação foram inapetência, sonolência, fadiga, dispneia e dor. 0 número de punções venosas periféricas foi superior ao número de hipodermóclises realizadas. Conclusão: os dados analisados permitiram caracterizar o perfil de pacientes oncológicos internados sob cuidados paliativos e a terapêutica adotada para a terapia medicamentosa. Pôde-se, ainda, identificar a baixa adesão da equipe à realização da hipodermóclise, o que remete à necessidade da realização de mais estudos com altos níveis de evidência para embasar a prática assistencial da equipe de enfermagem e contribuir para a qualidade de vida do paciente.
\end{abstract}

Descritores: Cuidados Paliativos; Hipodermóclise; Infusões Subcutâneas; Cateterismo Periférico; Enfermagem.

\section{ABSTRACT}

Objective: To characterize cancer patients hospitalized under palliative care who underwent peripheral venipuncture and hypodermoclysis according to sociodemographic and clinical variables. Method: This is an observational, descriptive, and prospective study. Interviews were carried out with the patients and/or their caregiver, as well as consultation of medical records, evaluation, and daily monitoring of the puncture. Results: 45 patients of both genders and of 18+ years of age participated in the study. The functional assessment of patients at admission was 30\%, indicating extremely disabled patients and in need of hospitalization. The most frequent symptoms presented by patients on the day of hospitalization were lack of appetite, drowsiness, fatigue, dyspnea, and pain. The number of peripheral venipunctures was higher than the number of hypodermoclyses performed. Conclusion: The analyzed data allowed to characterize the profile of cancer patients hospitalized under palliative care and the therapy adopted for drug therapy. It was also possible to identify the low adherence of the team to the performance of hypodermoclysis, which refers to the need for further studies with high levels of evidence to support the care practice of the nursing team and contribute to the patient's quality of life.

Descriptors: Palliative Care; Hypodermoclysis; Infusions, Subcutaneous; Catheterization, Peripheral; Nursing.

\section{RESUMEN}

Objetivo: caracterizar los pacientes con cáncer hospitalizados bajo cuidados paliativos, que se sometieron a venopunción periférica e hipodermoclisis de acuerdo con variables sociodemográficas y clínicas. Método: estudio observacional, descriptivo y prospectivo. Se realizaron entrevistas con el paciente y / o su cuidador, consulta de historias clínicas, evaluación y seguimiento diario de la punción. Resultados: 45 pacientes de ambos sexos y mayores de 18 años participaron en el estudio. La evaluación funcional de los pacientes al ingreso fue del $30 \%$, lo que indica pacientes extremadamente discapacitados y que necesitan hospitalización. Los síntomas más frecuentes que presentaron el día de la hospitalización fueron: falta de apetito, somnolencia, fatiga, disnea y dolor. El número de punciones venosas periféricas fue mayor que el número de hipodermoclisis realizado. Conclusión: los datos analizados permitieron caracterizar el perfil de pacientes con cáncer hospitalizados bajo cuidados paliativos y el tratamiento adoptado para la terapia farmacológica. También fue posible identificar la baja adhesión del equipo a la realización de la hipodermoclisis, lo que remite a la necesidad de realizar más estudios con altos niveles de evidencia para apoyar la práctica de atención del equipo de enfermería y contribuir a la calidad de vida del paciente.

Descriptores: Cuidados Paliativos; Hipodermoclisis; Infusiones Subcutáneas; Cateterismo Periférico; Enfermería.

${ }^{1}$ Enfermeira, Escola de Enfermagem de Ribeirão Preto da Universidade de São Paulo. ${ }^{2}$ Enfermeira, Escola de Enfermagem de Ribeirão Preto da Universidade de São Paulo. ${ }^{3}$ Enfermeira, Faculdade de Medicina, Universidade Estadual Paulista "Julio de Mesquita Filho". "Enfermeira, Doutora, Universidade Federal de Mato Grosso do Sul. ${ }^{5}$ Enfermeira, Doutora, Escola de Enfermagem de Ribeirão Preto da Universidade de São Paulo. ${ }^{6}$ Enfermeira, Doutora, Escola de Enfermagem de Ribeirão Preto da Universidade de São Paulo.

Como citar este artigo:

Moreira MR, Souza AC, Villar J, et al. Caracterização de pacientes sob cuidados paliativos submetidos à punção venosa periférica e à hipodermóclise. Revista de Enfermagem do Centro-Oeste Mineiro. 2020;10:e4032. [Access ; Available in: DOI: 


\section{INTRODUÇÃO}

Dentre as doenças crônicas não transmissíveis, o câncer tem ocupado lugar de destaque. Quando em fase avançada, a evolução da doença é acompanhada de sinais e sintomas de difícil controle, tais como, dor, náuseas, vômitos, anorexia, fadiga, depressão, ansiedade, constipação, entre outros ${ }^{(1)}$. Ainda, considerando o paciente oncológico em fase avançada, é comum a perda da funcionalidade somada às profundas alterações físicas, emocionais e espirituais experimentadas pelos pacientes, a partir do diagnóstico, até a morte ${ }^{(2)}$. Nesse sentido, a oferta de cuidados paliativos tem impacto positivo sobre o controle dos sintomas e a melhoria da qualidade de vida dos pacientes.

Dessa forma, é razoável que se discuta cada vez mais sobre os métodos alternativos, como o uso da via subcutânea para a terapia medicamentosa. Essa via é indicada para a administração de medicamentos e soluções de reidratação, quando as vias endovenosa e oral se apresentam limitadas e procedimentos invasivos estejam contraindicados ${ }^{(3)}$.

O uso da via subcutânea, por meio da realização da hipodermóclise, é considerado uma alternativa viável, segura e sem complicações graves, porém, ainda é uma técnica pouco conhecida e utilizada no cenário hospitalar, existindo questionamentos diversos quanto à realização da técnica, quais medicamentos e fluidos podem ser seguramente administrados, volume máximo e velocidade de infusão permitidos, entre outros aspectos ${ }^{(4-6)}$.

A hipodermóclise consiste na administração de medicamentos e fluídos no espaço subcutâneo, de forma contínua ou intermitente ${ }^{(3)}$. A via subcutânea é a possível via a ser utilizada, principalmente, em pacientes idosos e sob cuidados paliativos que, frequentemente, apresentam condições que impossibilitam a manutenção adequada de níveis de hidratação e nutrição, necessitando de vias alternativas para suporte clínico ${ }^{(7)}$.

Entretanto, na prática clínica atual, na assistência de enfermagem a pacientes em cuidados paliativos, a administração parenteral de medicamentos e soluções é feita, prioritariamente, pela via endovenosa, mesmo que, para a sua obtenção o paciente seja submetido a inúmeras punções e maior risco de infecção de corrente sanguínea, mesmo sendo, a via subcutânea, uma opção viável, segura, mais confortável e de menor custo. Ainda, a manutenção de um cateter venoso periférico requer um maior esforço e cuidado e o risco de complicações é significativo, comprometendo a segurança do paciente ${ }^{(8)}$.

Assim, neste estudo objetivou-se caracterizar os pacientes oncológicos internados sob cuidados paliativos submetidos a punção venosa periférica e a hipodermóclise, segundo as variáveis sociodemográficas e clínicas.

\section{MATERIAL E MÉTODO}

Trata-se de estudo observacional, de caráter descritivo e com seguimento prospectivo, realizado de janeiro a maio de 2019 , na enfermaria de clínica médica de um hospital universitário do interior paulista.

Uma amostra não probabilística e consecutiva foi constituída por 45 pacientes que atenderam aos seguintes critérios de inclusão: oncológicos, internados sob cuidados paliativos, de ambos os sexos, com idade superior a 18 anos e com necessidade de punção para terapia medicamentosa parenteral durante a internação.

A coleta de dados foi realizada, por meio de entrevista com o paciente ou seu responsável legal, consulta ao prontuário, avaliação e acompanhamento diário da punção (venosa ou subcutânea), desde sua obtenção até o momento em que não houve mais indicação (suspensão dos medicamentos parenterais, alta hospitalar ou óbito do paciente). Para esse estudo, foram utilizados questionários para a obtenção das variáveis sociodemográficas e clínicas; Karnofsky Performance Status $(\mathrm{KPS})^{(9)}$ para a avaliação funcional dos participantes; Escala de Avaliação de Sintomas de Edmonton - BR (ESAS-Br) ${ }^{(10)}$ para a identificação de sinais e sintomas apresentados pelos pacientes e roteiro para avaliação e acompanhamento diário da punção.

$\mathrm{O}$ projeto de pesquisa foi elaborado, de acordo com os preceitos da Resolução CNS $466 / 12^{(11)}$ e foi aprovado pelo Comitê de Ética em Pesquisa da Escola de Enfermagem de Ribeirão Preto da Universidade de São Paulo, sob o CAAE: 91320318.1.0000.5393 e número do parecer 2.907.347. Os pacientes foram convidados a participar da pesquisa e a coleta de dados só foi realizada após seu consentimento e respectiva assinatura do Termo de Consentimento Livre e Esclarecido.

Foram realizadas análises de frequência simples para variáveis nominais ou categóricas e análise de tendência central (média e mediana) e 
dispersão (desvio-padrão) para as variáveis contínuas.

\section{RESULTADOS E DISCUSSÃO}

A amostra deste estudo foi caracterizada, em sua maioria, por indivíduos do sexo masculino (62,22\%) e com idade média de 66,56 anos $(D P=14,01)$. Os diagnósticos oncológicos mais observados, neste estudo, foram câncer de pulmão e câncer colorretal correspondendo a $20,00 \%$ e $15,56 \%$, respectivamente e a comorbidade mais frequente apresentada pelos participantes foi a hipertensão arterial sistêmica (20; 44,44\%).

Dentre os participantes que apresentavam metástases, as mais frequentes foram hepática (8; $17,78 \%)$, pulmonar $(6 ; 13,33 \%)$ e óssea (5; $11,11 \%)$. No que se refere à terapia modificadora da doença implementada previamente à indicação de cuidados paliativos, $61,36 \%$ dos pacientes foram submetidos à quimioterapia e 56,82\%, à radioterapia.

Entre os sintomas apresentados pelos pacientes, no dia da internação, os mais observados foram inapetência (39; 86,60\%), sonolência (37; 82,20\%), fadiga (36; 80,00\%), dispneia $(24 ; 53,30 \%)$ e dor de moderada a forte intensidade $(21 ; 46,60 \%)$.

Os medicamentos mais utilizados pelos pacientes, durante a internação foram: dipirona (31; 15,12\%), morfina $(27 ; 13,17 \%)$, ondansetrona $(23 ; 11,21 \%)$ e dexametasona $(21 ; 10,24 \%)$.

Ao todo, foram realizadas 134 punções, nessa amostra, durante o período de internação. Verificou-se uma média de duas punções venosas periféricas por paciente e o número de hipodermóclises foi, substancialmente, inferior ao de punções venosas.

$$
\text { As demais características }
$$
sociodemográficas e clínicas dos pacientes estão descritas na Tabela 1.

Tabela 1 - Caracterização sociodemográfica e clínica dos pacientes oncológicos internados sob cuidados paliativos. Ribeirão Preto - SP, 2019. $(n=45)$.

\begin{tabular}{|c|c|c|}
\hline Variável & Média (DP) & $\mathrm{n}(\%)$ \\
\hline \multicolumn{3}{|l|}{ Sexo } \\
\hline Masculino & & $28(62,22)$ \\
\hline Feminino & & $17(37,78)$ \\
\hline Idade (anos) & $66,56(14,01)$ & \\
\hline \multicolumn{3}{|l|}{ Estado Civil } \\
\hline Casado/União Consensual & & $21(46,67)$ \\
\hline Solteiro & & $8(17,78)$ \\
\hline Separado/Divorciado & & $8(17,78)$ \\
\hline Viúvo & & $8(17,78)$ \\
\hline Escolaridade (anos) & $4,81(2,60)$ & \\
\hline \multicolumn{3}{|l|}{ Principal cuidador } \\
\hline Filho (a) & & $18(40,00)$ \\
\hline Esposa/marido/cônjuge & & $14(31,11)$ \\
\hline Outro cuidador familiar & & $12(26,67)$ \\
\hline Cuidador não familiar & & $1(2,22)$ \\
\hline Idade do cuidador (anos) & $47,62(16,85)$ & \\
\hline Escolaridade do cuidador & $8,02(4,10)$ & \\
\hline \multicolumn{3}{|l|}{ Doença oncológica de base } \\
\hline Câncer de pulmão & & $9(20)$ \\
\hline Câncer colorretal & & $7(15,56)$ \\
\hline Câncer de mama & & $5(11,11)$ \\
\hline Câncer de esôfago & & $4(8,89)$ \\
\hline \multicolumn{3}{|l|}{ Metástases } \\
\hline Hepática & & $8(17,78)$ \\
\hline Pulmonar & & $6(13,33)$ \\
\hline Óssea & & $5(11,11)$ \\
\hline Linfonodos & & $3(6,67)$ \\
\hline \multicolumn{3}{|c|}{ Tratamento modificador da doença } \\
\hline Quimioterapia & & $27(61,36)$ \\
\hline Radioterapia & & $25(56,82)$ \\
\hline Cirurgia & & $20(44,44)$ \\
\hline Virgem de tratamento & & $11(25,00)$ \\
\hline Hormonioterapia & & $2(4,55)$ \\
\hline
\end{tabular}




\begin{tabular}{ll}
\hline \hline Variável & Média (DP) \\
\hline KPS & $\mathbf{n}(\%)$ \\
$30 \%$ & $20(44,40)$ \\
$40 \%$ & $10(22,20)$ \\
$20 \%$ & $6(13,30)$ \\
$50 \%$ & $4(8,90)$ \\
$60 \%$ & $2(4,40)$ \\
$70 \%$ & $2(4,40)$ \\
$10 \%$ & $1(2,20)$ \\
\hline \hline
\end{tabular}

Fonte: dados do estudo.

Seguem, na Tabela 2, os dados relacionados às punções observadas.

Tabela 2 - Caracterização das punções a que os pacientes foram submetidos, durante a internação. Ribeirão Preto - SP, 2019. ( $n=134)$.

\begin{tabular}{lc}
\hline \multicolumn{1}{c}{ Variável } & $\mathbf{n}(\%)$ \\
\hline Tipo de punção & \\
$\quad$ Punção venosa periférica & $117(87,00)$ \\
Hipodermóclise & $17(13,00)$ \\
Finalidade da punção & \\
$\quad$ Analgesia & $23(30,00)$ \\
Antibioticoterapia & $23(30,00)$ \\
Tentativas de punção venosa para a & \\
obtenção de acesso venoso & \\
Uma & $42(41,00)$ \\
Duas & $23(22,00)$ \\
Três & $5(4,00)$ \\
Quatro & $4(3,00)$ \\
Cinco & $2(1,00)$ \\
Seis & $25(24,00)$ \\
Local da punção SC & \\
Região abdominal & $6(35,00)$ \\
Região anterolateral da coxa & $6(35,00)$ \\
Região deltoidea & $5(29,00)$ \\
\hline \hline
\end{tabular}

Fonte: dados do estudo.

Esse estudo buscou caracterizar os pacientes oncológicos internados sob cuidados paliativos submetidos a punção venosa periférica e a hipodermóclise, segundo as variáveis sociodemográficas e clínicas.

Nessa direção, este estudo identificou maior prevalência do sexo masculino. Esse resultado está em concordância com a literatura, a qual retrata que a incidência oncológica, em países em desenvolvimento no biênio 2018-2019, foi maior no sexo masculino, do que no sexo feminino, sendo as taxas de incidência correspondentes a 217,27/100 mil habitantes e $191,78 / 100$ mil, respectivamente ${ }^{(12)}$.

A idade dos participantes, deste estudo, variou de 22 a 93 anos, com média de 66 anos ( $D P=14)$. A literatura estima que, em 2030, a carga global será de 21,4 milhões de casos novos de câncer e 13,2 milhões de mortes por câncer, como resultado do crescimento e do envelhecimento da população, associado à redução de mortes por doenças infecciosas e da mortalidade infantil em países em desenvolvimento ${ }^{(13)}$.

O diagnóstico médico mais evidenciado, no estudo, foi câncer de pulmão (20,00\%), seguido do câncer colorretal $(15,56 \%)$ e câncer de mama feminino $(11,11 \%)$, diferindo, parcialmente, da literatura sobre o assunto, uma vez que, os estudos mostram que, em 2012, nos países em desenvolvimento, os tipos mais comuns de câncer masculino foram pulmão, estômago e fígado. No Brasil, os cânceres mais incidentes no sexo masculino são próstata, pulmão e colorretal, respectivamente. No entanto, o câncer de próstata possui perfil de mortalidade menor, com longa sobrevida. No que se refere à população feminina, a maior incidência foi de câncer de mama, colo do útero e pulmão. Em 2018, as estimativas de novos casos de câncer de pulmão foram 31.270, sendo 18.740 homens e 12.530 mulheres. O número de óbitos esperados em indivíduos com esse diagnóstico é de 27.931, sendo 16.139 homens e 11.792 mulheres ${ }^{(14-17)}$.

Este estudo mostra que a maior incidência de metástase foi hepática, sendo assim, até 35\% dos pacientes que vão a óbito por algum tipo de câncer, tendem a possuir metástase hepática, durante a progressão da doença e os tipos de câncer mais comuns que evoluem com metástase para o fígado são: carcinoma do pâncreas, carcinoma colorretal, carcinoma de estômago, carcinoma da mama, carcinoma do esôfago, carcinoma do pulmão e o tumor carcinoide ${ }^{(15)}$.

No que se refere à avaliação funcional do paciente na internação, o estudo identificou maior frequência de KPS de $30 \%$, o que corresponde a pacientes em fase avançada da doença, extremamente incapacitados e, embora a morte não fosse iminente, apresentavam necessidade de métodos alternativos para a redução do sofrimento ${ }^{(9)}$. Ou seja, o paciente oncológico em cuidado paliativo, neste estudo, internou em uma condição de saúde bastante comprometida. O prejuízo na capacidade funcional, decorrente do câncer e do avanço da doença, afeta a capacidade do paciente no desempenho das atividades da 
vida diária, interferindo em suas relações sociais, impactando, negativamente, sua qualidade de vida $^{(1)}$. Tais aspectos devem ser considerados na promoção de um cuidado humanizado a esse perfil de paciente durante a internação.

No que se refere à terapêutica adotada para a administração de medicamentos parenterais, este estudo identificou uma discrepância significativa entre o número de punções venosas periféricas $(87,00 \%)$ e hipodermóclises $(13,00 \%)$, ao longo da internação dos pacientes.

O número de tentativas para a obtenção de um acesso venoso periférico, chama atenção, sendo que a maioria foi obtida na primeira tentativa (41\%) e a segunda maior taxa de tentativas foi de seis vezes para a obtenção de um acesso venoso periférico. Tal fato está em desacordo com as recomendações preconizadas pela Agência Nacional de Vigilância Sanitária (ANVISA), que orienta limitar a duas tentativas de punção venosa periférica, por profissional de saúde e, no máximo, quatro tentativas por paciente ${ }^{(16)}$.

As múltiplas tentativas de punção, além de promover dor e desconforto ao paciente, tardam o início da infusão medicamentosa, prejudicam a rede venosa, além de tornarem os custos e riscos de complicações maiores. Ademais, os pacientes com rede venosa comprometida, como os participantes no presente estudo, devem ser submetidos a uma avaliação multidisciplinar cautelosa, a fim de se discutir as opções viáveis de acesso.

Do ponto de vista técnico, a punção subcutânea é mais fácil de ser realizada do que a intravenosa, assim como sua manutenção e custo e a opção por essa prática deve ser considerada quando o paciente apresenta difícil acesso venoso $^{(18)}$.

Observa-se que os profissionais de enfermagem optam pela via endovenosa, em detrimento à subcutânea, mesmo que o paciente seja submetido a inúmeras tentativas de punção venosa, comprometendo a qualidade de vida dos pacientes. Tais resultados podem estar relacionados ao fato de que a hipodermóclise é uma técnica ainda pouco difundida no meio profissional e acadêmico, no que concerne a pacientes sem condições de uso das vias de administração tradicionais para medicamentos e ou para reposição de soluções, como evidenciado na literatura ${ }^{(17,19-21)}$. Ainda, observam-se poucas evidências na literatura sobre os benefícios da hipodermóclise para esse perfil de pacientes ${ }^{(22)}$.
No presente estudo, os locais de escolha mais frequentes para a realização de hipodermóclise foram a região abdominal e a região anterolateral da coxa ( $35 \%$ cada). Estudos mostram que os locais mais utilizados para a realização da punção subcutânea são: a região torácica e a parede abdominal lateral. As regiões que permitem maior volume de infusão são região anterolateral da coxa, região abdominal e região intraescapular, sendo o volume máximo tolerado por cada sítio, $1500 \mathrm{ml}, 1000 \mathrm{ml} \mathrm{e} 1000 \mathrm{ml}$ em 24h, respectivamente. Vale ressaltar que o volume total infundido por via subcutânea não deve ultrapassar $3000 \mathrm{ml}$ em $24 \mathrm{~h}^{(8)}$.

As principais finalidades das punções observadas foram: analgesia e antibioticoterapia e os medicamentos mais administrados: a dipirona $(31,00 \%)$, morfina $(27,00 \%)$ e ondansetrona $(23,00 \%)$. Os antibióticos, de modo geral, corresponderam a 15,12\% dos medicamentos mais prescritos. Tais resultados vão ao encontro da literatura sobre o tema ao identificar que a principal indicação de punção para pacientes oncológicos em cuidados paliativos é o alívio de sintomas provenientes da progressão da doença ${ }^{(4,8)}$.

Ainda, no que se refere aos sinais e sintomas apresentados pelos pacientes do estudo no dia da internação, observamos a elevada frequência de sintomas desconfortáveis para o paciente, tais como, inapetência, sonolência, fadiga, dispneia e dor, comprometendo sua qualidade de vida e conforto. O controle de sintomas constitui um importante desafio para a equipe de saúde na assistência a pacientes com câncer avançado e, por vezes, seu difícil manejo impacta diretamente a qualidade de vida dos pacientes $^{(23)}$.

\section{CONCLUSÃO}

Os dados analisados permitiram caracterizar o perfil de pacientes oncológicos internados sob cuidados paliativos e a terapêutica adotada para a terapia medicamentosa. Dessa forma, a importância do estudo está relacionada à possibilidade de planejamento da melhor estratégia para a obtenção de uma via para a administração de medicamentos parenterais, de acordo com as limitações e necessidades apresentadas por esse perfil de paciente, contribuindo, sobremaneira, para a qualidade de vida do paciente em cuidados paliativos, durante a internação hospitalar. Ou seja, conhecer o perfil dos pacientes oncológicos sob cuidados paliativos 
e candidatos a uma punção para terapia medicamentosa permite aos profissionais levar tais aspectos em consideração, na opção pela escolha da via mais adequada, considerando seu perfil, de modo a minimizar os desconfortos inerentes a cada técnica e, com isso, promover sua qualidade de vida.

Ainda, neste estudo, pôde-se identificar a baixa adesão da equipe à realização da hipodermóclise, fato que pode estar relacionado à falta de conhecimento sobre a técnica pelos profissionais de saúde, poucas evidências publicadas sobre seus benefícios e a técnica em si. Dessa forma, faz-se necessária a realização de mais estudos com altos níveis de evidência para embasar a prática assistencial da equipe de enfermagem, favorecendo o uso da via subcutânea, especialmente, entre os pacientes com perfil semelhante ao dos pacientes deste estudo, contribuindo, assim, para sua melhor qualidade de vida.

Uma das limitações do estudo refere-se ao baixo número de hipodermóclises observadas no período do estudo, o que impediu a realização de alguns tipos de análises, tais como: a associação entre o tipo de punção e a funcionalidade do paciente, o que permitiria avaliar se pacientes com pior funcionalidade seriam mais submetidos à punção subcutânea, fato que, na prática clínica, é possível observar, porém sem comprovação científica.

\section{REFERÊNCIAS}

1- Freire MEM, Costa SFG, Lima RAG, Sawada NO. Qualidade de vida relacionada à saúde de pacientes com câncer em cuidados paliativos. Texto Contexto-Enferm. 2018;27(2):1-13. DOI: 10.1590/0104-070720180005420016

2- Minosso JSM, Souza LJ, Oliveira MAC. Reabilitação em cuidados paliativos. Texto Contexto-Enferm. 2016; 25(3):1-9. DOI: 10.1590/0104-07072016001470015

3- Scales K. Use of hypodermoclysis to manage dehydration. Nurs Older People 2011;23(5):16-22. DOI: 10.7748/nop2011.06.23.5.16.c8528

4- Bruno VG. Hipodermóclise: Revisão de literatura para auxiliar a prática clínica. Einstein 2015;13(1):122-8. DOI: $\quad$ 10.1590/S167945082015RW2572

5- Urbanetto JS, Peixoto CG, May TA. Incidence of phlebitis associated with the use of peripheral IV catheter and following catheter removal. Rev Latino-Am Enfermagem 2016;24:1-8. DOI: 10.1590/1518-8345.0604.2746
6- Nunes PMSA, Souza, RCS. Efeitos adversos da hipodermóclise em pacientes adultos: Revisão integrativa. Rev Min Enferm. 2016;20:1-6. DOI: 10.5935/1415-2762.20160020

7- Ministério da Saúde (BR). Terapia subcutânea no câncer avançado. Rio de Janeiro: INCA; 2009.

8- Azevedo DL. O uso da via subcutânea em geriatria e cuidados paliativos: Um guia da SBGG e da ANCP para profissionais. 2a ed. Rio de Janeiro: SBGG; 2017.

9- Karnofsky DA, Burchenal JH. The clinical evaluation of chemotherapeutic agents in cancer. In McCleod CM. The clinical evaluation of chemotherapeutic agents. New York: Columbia University Press; 1949. p. 191-205.

10- Paiva CE, Manfredi NILL, Paiva BSR, Hui D, Bruera $\mathrm{E}$. The brazilian version of the Edmonton Symptom Assessment System (ESAS) is a feasible, valid and reliable instrument for the measurement of symptoms in advanced cancer patients. PLoS One 2015;10(7):1-13. DOI: 10.1371/journal.pone.0132073

11- Brasil. Conselho Nacional de Saúde. Resolução no 466, de 12 de dezembro de 2012. Diário Oficial da União 2013;12(1):59.

12- Brasil. Ministério da Saúde. Incidência de câncer no Brasil. Rio de Janeiro: INCA; 2018.

13- Brasil. Ministério da Saúde. Instituto Nacional de Câncer. Incidência de câncer no Brasil. Rio de Janeiro: INCA; 2014.

14- Brasil. Sistema de informação de mortalidade. Portal da Saúde 2017. [citado em 22 ago 2020]. Acesso em: http://www2.datasus.gov.br/DATASUS/

15- Brasil. Ministério da Saúde. Câncer de fígado. Rio de Janeiro: INCA; 2019.

16- Brasil. Agência Nacional de Vigilância Sanitária. Medidas de prevenção de infecção relacionada à assistência à saúde. Brasília: Anvisa; 2017.

17- Veras GL, Faustino AM, Reis PED, Paula G, Simino R. Evidências clínicas no uso da hipodermóclise em pacientes oncológicos. Gest Saúde 2014 [citado em 7 ago 2019]; 5(nesp):287793.

Acesso

em:

https://periodicos.unb.br/index.php/rgs/article/v iew/1591

18- Sasson M, Shvasrzman P. Hypodermoclysis: An alternative infusion technique. Am Fam Physician. 2001 [citado em 7 ago 2019]; 64:15758. Acesso

em: https://pdfs.semanticscholar.org/6484/49e37d6a 22be810ae5b423fce2a389bbcd6b.pdf 
19- Araújo AS, Mota LM. Uma alternativa do passado com futuro: Hipodermóclise, uma revisão integrativa. Interfaces Científicas - Saúde e Ambiente 2014;2(3):45-51. DOI: https://doi.org/10.17564/2316-

\subsection{4v2n3p45-51}

20- Zironde ES, Marzenini NL, Soler VM. Hipodermóclise: Redescoberta da via subcutânea para o tratamento de indivíduos vulneráveis. CuidArte, Enferm. 2014 [citado em 22 ago 2020]; 8(1):55-61. Acesso em: https://pesquisa.bvsalud.org/portal/resource/pt/ $\underline{\text { bde- } 25773}$

21- Cardoso DH, Mortola LA, Arrieira ICO. Terapia subcutânea para pacientes em cuidados paliativos: A experiência de enfermeiras na atenção domiciliar. J Nurs Health 2016;6(2):34654. DOI: $10.15210 /$ jonah.v6i2.6478

22- Braz CL, Pereira RCC, Costa JM. Administração de medicamentos por hipodermóclise: Uma revisão da literatura. Rev Bras Farm Hosp Serv. 2015 [citado em 22 ago 2020]; 6(1):6-12. Acesso em:

https://www.rbfhss.org.br/sbrafh/article/view/2 $\underline{17 / 218}$

23- Currow DC, Clack K, Kamal A, Collier A, Agar $M R$, Lovel MR, et al. The population burden of chronic symptoms that substantially predate the diagnosis of a Life-Limiting Illness. J Palliat Med. 2015;18(6):480-5. DOI: 10.1089/ipm.2014.0444

Nota: este estudo faz parte do Trabalho de Conclusão de Curso intitulado: "Caracterização de pacientes sob cuidados paliativos submetidos à punção venosa periférica e à hipodermóclise" utilizado para cumprimento das exigências necessárias para obtenção do título de Bacharel em Enfermagem da Escola de Enfermagem de Ribeirão Preto (EERP) da Universidade de São Paulo (USP), e com fomento pelo Programa Unificado de Bolsas (PUB) que integra a Política de Apoio à Permanência e Formação Estudantil da USP, com o objetivo de contribuir para a formação acadêmica e profissional.

Recebido em: 30/09/2020

Aprovado em: 18/12/2020

\section{Endereço de correspondência:}

University of São Paulo at Ribeirão Preto, College of Nursing WHO Collaborating Centre for Nursing Research

Fone/Phone: 550XX(16) 3315-3401

Email: fbolela@usp.br 